\title{
Gender Differences in Motor Coordination and Physical Activity
}

\author{
Riza Adriyani*, Dody Iskandar, Lia Siti Camelia \\ Balai Kesehatan Olahraga Masyarakat Bandung \\ Ministry of Health, Indonesia \\ Bandung, Indonesia \\ *bkom_bandung@yahoo.com
}

\begin{abstract}
The research highlights gender differences in gross motor coordination. Preadolescence is the best time for motor skill interventions. Good motor coordination indicates an increased physical activity in children. The purpose of this study was to know the differences of motor coordination and physical activity levels based on gender. School children consisted of 95 males (10.57 years) and 105 females (10.36 years) participated in this study. Weight status, height, and percentage of body fat were objectively measured by using standardized protocols to compare anthropometric characteristics. Körperkoordinations Test für Kinder (KTK) was used to assess gross motor coordination, while physical activity levels were assessed by Physical Activity Questionnaire for Children. Statistical analysis used an independent sample t-test (Mann Whitney $U$ test if not normally distributed) to analyse gender differences. The results showed that body mass index was almost similar in both groups. There were significant differences in motor coordination between boys and girls $(p<0.05)$. The mean motor quotients KTK (MQ KTK) of boys (83.34) was significantly higher than girls (72.39). Boys outperformed girls on hopping height, moving sideways, and jumping sideways. Only for walking backward girls were similar to boys. Physical activity was significantly different in both gender $(p<0.05)$. Girls were less active than boys. There were gender differences in gross motor coordination and physical activity levels in children. There is a need for better motor skill interventions in girls.
\end{abstract}

Keywords: gross coordination, activity levels, gender

\section{INTRODUCTION}

Physical activity has a crucial role to maintain our health. Improvement of physical fitness could be reached through regular participation in various physical activities. Many studies in developed countries concluded that physical activity declines in children of all ages [1,2]. In Indonesia, one in four residents had sedentary behaviour. Around $71.8 \%$ of children aged 10-14 years had sedentary behaviour for more than three hours/day. In middle childhood (6-9 years) and late childhood (10-13 years), better motor coordination could increase children's participation in many physical activities and games $[3,4]$. Motor coordination can be affected by many factors, such as involvement in organized sports education, the influence of environment and also family lifestyle. Motor coordination does not only have positive trajectories on physical activity but also promotes cognitive function and academic achievement in children [5]. Previous research showed that motor coordination was the best predictor for physical activity levels in children aged 6 to 10 years [6]. Children with better motor coordination could be more physically active in later life compared to those with poor motor coordination [7]. At school, children's motor coordination skills can develop better through various playful activities at recess time and high participation in physical education class [8]. Active transportation (walking and cycling) to school also had a positive relationship with total physical activity in boys [9]. Motor coordination had an inverse relationship with body mass index [8]. Several studies assessing gross motor coordination found impaired skills in obese children [10,11]. Motor coordination is not only correlated with health-related fitness, but also with sports with specific skills and daily physical activity [12]. Boys tend to be more active in their daily life than girls $[13,14]$. Girls tend to have lower habitual physical activity levels because lacking environmental (school, home and neighbourhood) supports $[8,13]$ and lower participation in organized sports $[8,13,15]$. The decline in gross motor coordination is in line with decreased physical activity levels. Walking backward and moving sideways significantly worse than age and genderspecific motor quotients norms [16]. Since motor coordination is associated with higher physical activity, boys were suggested to have a better motor coordination level than girls $[17,18]$. Although the gender differences in gross motor coordination and physical activity are well documented in high- income countries, there is still a limited number of researches in middle-income countries. Therefore, we want to find out if there are significant differences in motor coordination and physical activity levels by gender.

\section{METHOD}

\section{A. Participants}

Before collecting data, we informed the aim of the research to the headmaster, parents, and also class teachers. The children involved in sports clubs outside the school or obese were not recruited in this study. A total of 200 children (boys: $n$ =95; girls: $\mathrm{n}=105$ ) whose parents provided informed consent, participated in this research. The mean age of boys was $(10.57$ \pm 0.50 years $)$ while girls were $(10.36 \pm 0.48$ years $)$. The Research Ethics Committee, Ministry of Health-National Institute of Health Research and Development (19/07/2017) approved this study. 


\section{B. Experimental Protocol}

All participants completed the entire protocol in a single session. They have to warm-up and cool-down before and after performing the test. Obese children were excluded from this present study to ensure that body mass index did not affect motor coordination abilities.

1) Body mass index: Body height and body weight were measured using ZT 120 health scale. The weight was recorded to the nearest $0,1 \mathrm{~kg}$ and the height was also recorded to the nearest $0,01 \mathrm{~m}$. Body mass index was calculated for each subject by dividing the weight by the square of the height.

2) Body fat percentage: The measurement of body fat was using skinfold. It was located on triceps and calf (Fig. 1). We calculated the percentage of body fat applying the formula referenced in the Slaughter Lohman Formula manual [19]. The mean score of the three trials was determined.
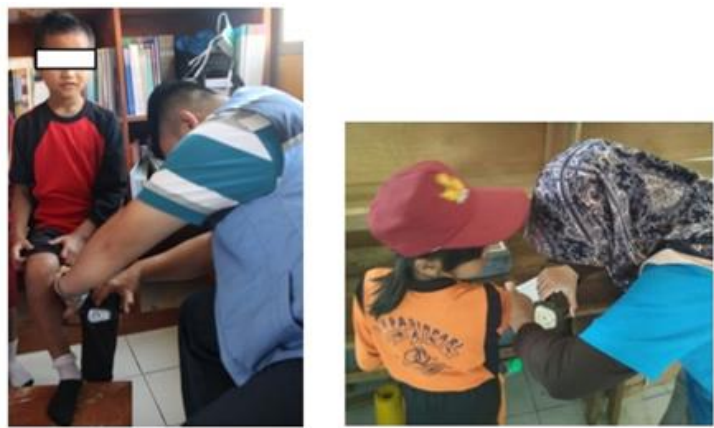

Fig. 1. Measurement site of body fat percentage on triceps and calf.

3) Motor coordination: We used Körperkoordinations Test für Kinder (KTK) by Kiphard and Schilling [20] which consisted of four subtests (Fig. 2):

- Walking Backwards (WB): walking backward three times along each of three balance beams ( 3 m length; $6,4.5$ and $3 \mathrm{~cm}$ width). A maximum of 24 steps (eight per trial) were counted for each balance beam, which comprises a maximum of 72 steps for this test. The score was converted to the motor quotient (MQ) result. Before the test, the subjects had one practice walk forward and backward on every balance beam.

- Hopping Height (HH): jumping from one leg over an increasing pile of pillows ( $40 \mathrm{~cm} \times 20 \mathrm{~cm} \times 5 \mathrm{~cm}$ each) after a short run-up. Every trial was evaluated and the subject had three trials on every height. Three, two or one point(s) were/was awarded for successful performance on the first, second or third trials, respectively. A maximum of 39 points could be scored for each leg, yielding a possible maximum score of 78 and then converted to the MQ result.

- Jumping Sideways (JS): jumping bilaterally as many times as possible over a wooden rod $(60 \mathrm{~cm} \times 4 \mathrm{~cm} \times 2$ $\mathrm{cm})$ in 15 seconds. The number of jumps over two trials was summed and then converted to the MQ result.
- Moving Sideways (MS): moving one plate on sideways and the subjects stood on the other plate $(25$ $\mathrm{cm} \times 25 \mathrm{~cm} \times 5 \mathrm{~cm}$ ) in 20 seconds. The plate had to be moved with two hands. The total amount of points was counted and summed over two trials, then converted to the MQ result.

The raw scores of each subtest were transformed into gender and age-specific MQ values according to the classification of Kiphard and Schilling. There are five categories: severe gross motor coordination disorder (MQ 5670), moderate gross motor coordination disorder (MQ 71-85), normal gross motor coordination (MQ 86-115), good gross motor coordination (MQ 116-130), and high gross motor coordination (MQ 131-145).

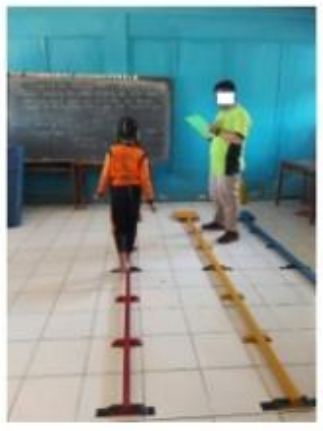

Fig 2 (a)

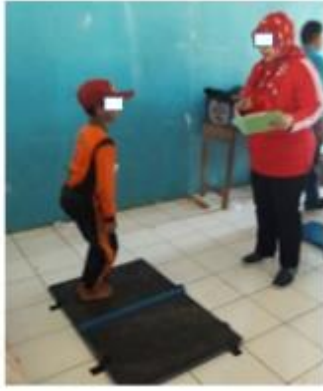

Fig 2 (c)

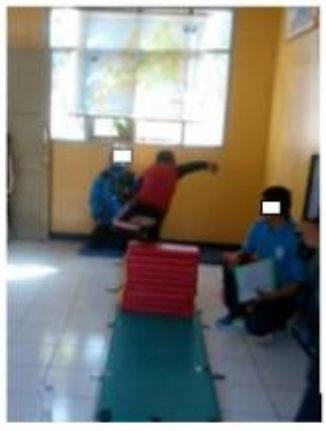

Fig 2 (b)

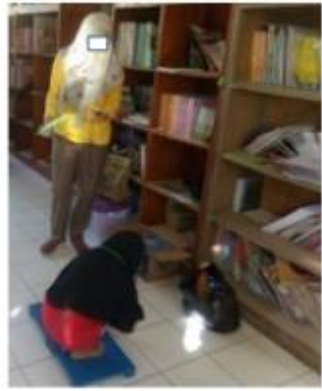

Fig 2 (d)
Fig. 2. The KTK test; walking backward (a) hopping height (b) jumping sideways (c) moving sideways (d).

4) Physical activity: Physical activity (PA) was assessed using a Physical Activity Questionnaire for Children (PAQ-C) that has high validity and moderate reliability [21]. The physical activity questionnaire for children is a selfadministered, 7-day recall instrument. It could assess general levels of physical activity throughout the school children (8-14 years). This instrument assesses a child's self-report of activity level in different settings and different times of the day (e.g. physical education class, activity at recess time, activity after school and also on the weekend). It provides a summary of the physical activity score derived from nine items, each scores a 5-point scale. 


\section{Statistical Analysis}

Normally the data were determined through the Kolmogorov-Smirnov test with $\mathrm{p}>0.05$. We used a parametric test or nonparametric test which was based on data distribution. Since the value of the subtests KTK - walking backward, jumping sideways did not follow a normal distribution, the non-parametric analysis was chosen. The comparison of motor coordination between genders was performed by independent sample t-test while the physical activity differences used Mann Whitney $\mathrm{U}$ test, with $\mathrm{p}$ value less than 0.05 considered significant. The data shown are means and percentages.

\section{RESULtS}

There were no significant differences in body mass index between boys and girls. However, body fat percentage was shown significantly different $(\mathrm{p}<0.05)$ in both groups (Table $1)$.

TABLE I. PARTICIPANTS ANTHROPOMETRIC CHARACTERISTICS

\begin{tabular}{|l|c|c|c|}
\hline General Anthropometric & Boys & Girls & $\boldsymbol{p}$ \\
\hline $\begin{array}{l}\text { Age } \\
(\text { Mean } \pm \text { SD) Years }\end{array}$ & $10.57 \pm 0.50$ & $10.36 \pm 0.48$ & \\
\hline $\begin{array}{l}\text { Height } \\
(\text { Mean } \pm \text { SD) Cm }\end{array}$ & $132.50 \pm 5.61$ & $133.86 \pm 6.51$ & \\
\hline Weight (Mean \pm SD) Kg & $29.68 \pm 4.87$ & $28.61 \pm 5.35$ & 0.33 \\
\hline $\begin{array}{l}\text { BMI } \\
(M e a n \pm S D) ~ K g / m^{2}\end{array}$ & $16.36 \pm 2.05$ & $16.63 \pm 1.84$ & $0.00 *$ \\
\hline $\begin{array}{l}\text { Body Fat Percentage } \\
(\text { Mean } \pm \text { SD) \% }\end{array}$ & $16.92 \pm 5.39$ & $20.95 \pm 4.35$ & *Significantly different $p$ value $<0.05$ \\
\hline
\end{tabular}

Based on motor coordination competence, there were significant differences in gross motor coordination performances between boys and girls. It showed significant differences in hopping height, jumping sideways and moving sideways (Table 2). The mean gross motor coordination of Indonesian children was lower than that of Belgium children [16] and German children [20]. The percentage distribution of motor quotients scores between boys and girls is shown in Fig.3. Children with motor problems were found in boys $(62.1 \%)$ and girls $(96.19 \%)$. Most of both groups had moderate motor disorder.

TABLE II. MOTOR COORDINATION DIFFERENCES BETWEEN BOYS AND GIRLS

\begin{tabular}{|l|l|l|l|}
\hline \multicolumn{1}{|c|}{$\begin{array}{c}\text { Motor } \\
\text { Coordination }\end{array}$} & \multicolumn{1}{|c|}{ Boys } & Girls & $\boldsymbol{p}$ \\
\hline MQ KTK & $83.34 \pm 8.45$ & $72.39 \pm 8.94$ & $0.00^{*}$ \\
\hline Walking backwards & $111.09 \pm 8.82$ & $108.94 \pm 8.85$ & 0.06 \\
\hline Hopping height & $91.84 \pm 9.80$ & $79.72 \pm 10.37$ & $0.00^{*}$ \\
\hline Jumping sideways & $98.51 \pm 13.62$ & $81.28 \pm 13.13$ & $0.00^{*}$ \\
\hline Moving sideways & $47.23 \pm 4.07$ & $45.04 \pm 4.05$ & $0.00^{*}$ \\
\hline
\end{tabular}

*Significantly different $p$ value $<0.05$

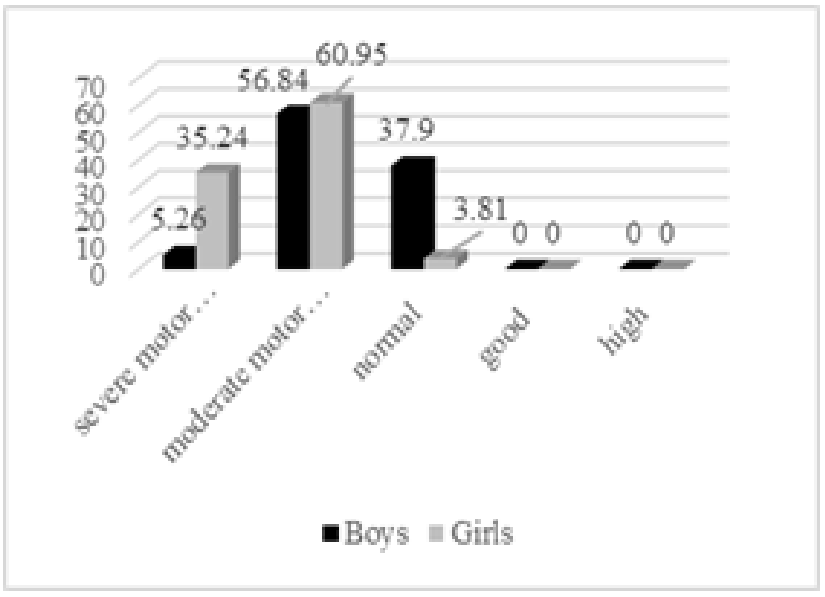

Fig. 3. Distribution of motor quotients between boys and girls.

There were significant differences on physical activity levels between boys and girls (Table 3 ). Boys were more active inside and outside the school rather than the girls.

TABLE III. PHYSICAL ACTIVITY DIFFERENCES BETWEEN BOYS AND GIRLS

\begin{tabular}{|l|l|l|l|}
\hline \multicolumn{1}{|c|}{$\begin{array}{c}\text { Physical } \\
\text { Activity }\end{array}$} & \multicolumn{1}{c|}{ Boys } & \multicolumn{1}{c|}{ Girls } & $\boldsymbol{p}$ \\
\hline Overall PAQ-C & $21.30 \pm 4.93$ & $18.07 \pm 4.25$ & $0.00^{*}$ \\
\hline $\begin{array}{l}\text { Physical } \\
\text { education class }\end{array}$ & $4.02 \pm 0.77$ & $3.67 \pm 0.88$ & $0.00^{*}$ \\
\hline Recess time & $3.31 \pm 1.01$ & $2.63 \pm 1.09$ & $0.00^{*}$ \\
\hline After school & $2.42 \pm 1.38$ & $1.98 \pm 1.28$ & $0.02^{*}$ \\
\hline Evening & $2.77 \pm 1.22$ & $2.09 \pm 1.08$ & $0.00^{*}$ \\
\hline Weekend & $2.34 \pm 1.03$ & $1.99 \pm 0.74$ & $0.01^{*}$ \\
\hline
\end{tabular}

\section{DISCUSSION}

This study was conducted to investigate whether there were still gender differences in children's body composition, physical activity and also gross motor coordination. To the best of our knowledge, there are still few studies about gross motor coordination in children using the KTK test in Indonesia. Körperkoordinations Test für Kinder is used to assess locomotor function and dynamic stability in children aged 5-14 years.

The findings that both groups had no different body mass index gave the fact that our participant's motor coordination abilities weren't influenced by weight status. Our results showed that there was a significant difference between boys and girls in their gross motor coordination, as measured by the total KTK MQ scores. This result is similar to those reported in other studies $[16,17]$ that there were significant differences in locomotor skills between gender.

There is a decline in gross motor coordination both in boys and girls compared to a study conducted by Vandorpe [16] and Ruzbarska [17]. None of both groups had better ("good" and "high") motor coordination. This study showed that Indonesian children are at greater risk of being less physically active, which in turn will affect their health status. 
The mean gross motor coordination of Indonesian children was lower than that of Belgium children [16] and German children [20]. Motor coordination is important for promoting physical activity and fitness throughout adulthood [3] which means it plays a crucial role for the health in children [22]. Children with poorer motor coordination are not only at the risk of being physically inactive $[22,23]$ but also have cognitive function and social interaction problems [24,25]. Thus, we need to take intervention in the development of motor coordination through practicing more skills and high participation in various activities [26-29].

Walking backwards (WB) and moving sideways (MS) are less strength-oriented subtests, while the other two tests, hopping height $(\mathrm{HH})$ and jumping sideways (JS), need more strength and endurance [30]. WB and MS are primary coordination and tend to be consistent over time. We found out that there were significant differences in body fat percentage between boys and girls. Boys had more muscle mass rather than fat mass, so boys had better strength and endurance than girls. A present study reveals that there is a significant difference in hopping height, jumping sideways and moving sideways performance between boys and girls. Our finding is almost similar to a study by Vandorpe [16] and Ruzbarska [17] that boys had better motor coordination abilities. No difference was observed between boys and girls for walking backwards. Boys have greater postural sway than girls [31], so it might affect their walking backwards performance test.

Dollman [32] showed that physical activity (active transport, physical education classes, organized sports) tends to decline nowadays. It might be caused by demographic influences, media used, and also school regulations. Our selfreported activities showed differences between boys and girls. The result of this study is in agreement with Nielsen et al. [33] and Field et al. [15] that boys played outdoors (after school, in the evening and also in the weekend) more frequently than girls, whereas girls performed sedentary activities. In accordance with Nielsen et al. [33], boys were also more active at school (e.q. physical education class and at recess time) compared to girls. Differences between boys and girls in performing their daily activities are presented in Table 3 . Not only inside the school but also outside the school that the girls were less active than boys. The result of this study is in agreement with Ishii et al. [13] and Telford et al. [14]. Low physical activity in girls might be influenced by socioecological factors at the individual, parents support, school and also environmental levels. Besides that, girls tend to engage with informal activities while boys tend to involve in organized sport activities.

According to Robinson et al. [4], motor coordination is a predictor of physical activity level in children, where children with high physical activity levels will have better a motor coordination level. In this study, we found out that boys had better physical activity levels than girls. Thus, boys also had better gross motor coordination compared to girls. Similar findings have been demonstrated in Australian children where gross motor skills had a positive relationship with an increased amount of physical activity at lunchtime, recess, and also after school [34].
We need to pay more attention to the development of children's motor skills in their everyday activities to increase their motor abilities, which will, in turn, increase their health and physical activity participation throughout adulthood. At 10 years, children need to participate in organized sports that involve specific sport skills and agility which can increase their motor coordination skills [35-37].

\section{CONCLUSION}

The findings of this study evidence that boys showed higher physical activity and motor coordination levels compared to girls. There is still a significant difference in gross motor coordination and physical activity levels by gender.

\section{REFERENCES}

[1] L. Basterfield, M.S. Pearce, A.J. Adamson, J.K. Frary, K.N. Parkinson, C.M. Wright, "Physical activity, sedentary behavior, and adiposity in English children,” Am J Prev Med, vol. 42(5), pp. 445-451, 2012.

[2] M.W. Beets, D. Bornstein, A. Beighle, B.J. Cardinal, C.F. Morgan, "Pedometer-measured physical activity patterns of youth: a 13-country review," Am J Prev Med, vol. 38(2), pp. 208-216, 2010.

[3] D.F. Stodden, J.D. Goodway, S.J. Langendorfer, M.A. Roberton, M.E Rudisill, C. Garcia, L.E. Garciaa, "Developmental perspective on the role of motor skill competence in physical activity: An emergent relationship," Quest, vol. 60, pp. 290-306, 2008.

[4] L.E. Robinson, D.F. Stodden, L.M. Barnett, V.P. Lopes, S.W. Logan, L. P. Rodrigues, E. D' Hondt, "Motor competence and its effect on positive developmental trajectories of health," Sports Med, Springer International Publishing Switzerland, 2015.

[5] R.F. Valter, L.S. Michelle, M. Thais, "Motor coordination correlates with academic achievement and cognitive function in children," Frontiers in Psychology, vol. 7, p. 318, 2016.

[6] V.P. Lopes, L.P. Rodrigues, J.A. Maia, R.M. Malina, "Motor coordination as predictor of physical activity in childhood," Scand J Med Sci Sports, vol. 21(5), pp.663-669, 2011.

[7] B.H. Wrotniak, L.H. Epstein, J.M. Dorn, K.E. Jones, V.A. Kondilis, "The relationship between motor proficiency and physical activity in children," Pediatrics, vol. 118(6), pp. e1758-e1765, 2006

[8] R. Chaves, A.B. Jones, T. Gomes, M. Souza, J. Maia, S. Pereira, “ Effects of individual and school-level characteristics on a child's gross motor coordination development," Int. J. Environ. Res, Public Health, vol. 12, pp. 8883-8896, 2015.

[9] S. Schoeppe, M.J. Duncan, H.M. Badland, M. Oliver, M. Browne, “ Associations between children's independent mobility and physical activity," BMC Public Health, vol.14, p. 91, 2014.

[10] V.P. Lopes, D.F. Stodden, M.M. Bianchi, J.A Maia, L.P. Rodriques, "Correlation between BMI and motor coordination in children," J Sci Med Sport, vol. 15(1), pp. 38-43, 2012.

[11] E. D’Hondt, B. Deforche, I. Gentler, Bourdeaudhuij, R. Veyens, R. Philippaerts, M. Lenoir, "A longitudinal analysis of gross motor coordination in overweight and obese children versus normal-weight peers," International Journal of Obesity, vol. 37, pp. 61-67, 2013.

[12] L. Lopes, R. Santos, C. Moreira, B. Pereira, V.P. Lopes, "Sensitivity and specificity of different measures of adiposity to distinguish between low/high motor coordination,” J Pediatr, vol. 91, pp. 44-51, 2015.

[13] K. Ishii, A. Shibata, M. Adachi, K. Nonoue, K. Oka, "Gender and grade differences in objectively measured physical activity and sedentary behavior patterns among Japanese children and adolescents: a crosssectional study," BMC Public Health, vol. 15, p.1254, 2015.

[14] R.M. Telford, R.D. Telford, L.S.Olive, T. Cochrane, R. Davey, "Why are girls less physically active than boys? Findings from the LOOK longitudinal study," PLoS ONE, vol. 11(3): e0150041. doi:10.1371/journal.pone.0150041, 2016. 
[28] A. Zask, L.M. Barnett, L. Rose, "Three year follow-up of an early childhood intervention: is movement skill sustained?," Int J Behav Nutr Phys Act, vol. 9, pp. 1-9, 2012.

[29] S.K. Lai, S.A. Costigan, P.J. Morgan, "Do school-based interventions focusing on physical activity, fitness, or fundamental movement skill competency produce a sustained impact in these outcomes in children and adolescents? A systematic review of follow-up studies," Sports Med, vol. 44, pp. 67-79, 2014.

[30] B. Pratorius, T.L. Milani, “ Motor abilities of children: abilities of coordination and balance: examination of differences between children of different social groups," Deut Z Sportmed, vol. 55, pp. 172-176, 2004.

[31] K.J. Mickle, B.J. Munro, J.R. Steele, "Gender and age affect balance performance in primary school-aged children," Journal of Science and Medicine in Sports, vol. 14, pp. 243-248, 2011.

[32] J. Dollman, K. Norton, L. Norton, V. Cleland, "Evidence for secular trends in children's physical activity behavior," Br J Sports Med, vol. 39(12), pp. 892-897, 2005.

[33] G. Nielsen, G. Pfister, L.B. Andersen, "Gender differences in the daily physical activities of Danish school children," European Physical Education Review, vol. 17(1), pp. 69-90, 2011.

[34] K.E. Cohen, P.J. Morgan, R.C. Plotnikoff, R. Callister, D.R. Lubans, "Fundamental movement skills and physical activity among children living in low-income communities: a cross-sectional study," Int J Behav Nutr Phys Act, vol. 11(1), p. 49, 2014.

[35] W.B. Strong, R.M. Maline, C.J. Blimkie, S.R. Daniels, R.K. Dishman, B. Gutin, F. Trudeau, "Evidence based physical activity for school-age youth," Journal of Pediatrics, vol. 146(6), pp. 732-737, 2005.

[36] P.D. Loprinzi, R.E. Davis, Y. Chieh Fu, "Early motor skill competence as a mediator of child and adult physical activity," Preventive Medicine Reports, vol. 2, pp. 833-838, 2015.

[37] S.S. Geertsen, R. Thomas, M.N. Larsen, J.N. Andersen, M.K. Jensen, V. Korup, C.M. Nielsen, J. Wienecke, C. Ritz, P. Krustrup, J. Lundbyejensen, I.M. Dahn, "Motor skills and exercise capacity are associated with objective measures of cognitive functions and academic performance in preadolescent children.” Plos ONE, pp. 1-16, 2016. 